\title{
CAFCLA: An AmI-Based Framework to Design and Develop Context-Aware Collaborative Learning Activities
}

\author{
Óscar García, Ricardo S. Alonso, Dante I. Tapia, and Juan M. Corchado \\ Department of Computer Science and Automation, \\ University of Salamanca, Plaza de la Merced, s/n, 37008, Salamanca, Spain \\ \{oscgar, ralorin, dantetapia, corchado\} @usal.es
}

\begin{abstract}
Ambient Intelligence (AmI) promotes the integration of Information and Communication Technologies (ICT) in daily life in order to ease the execution of everyday tasks. In this sense, education becomes a field where AmI can improve the learning process by means of context-aware technologies. However, it is necessary to develop new tools that can be adapted to a wide range of technologies and application scenarios. Here is where Agent Technology can demonstrate its potential. This paper presents CAFCLA, a multi-agent framework that allows developing learning applications based on the pedagogical CSCL (Computer Supported Collaborative Learning) approach and the Ambient Intelligence paradigm. CAFCLA integrates different context-aware technologies, so that learning applications designed, developed and deployed upon it are dynamic, adaptive and easy to use by users such as students and teachers.
\end{abstract}

Keywords: Ambient Intelligence, Mobile technologies, Computer Supported Collaborative Learning, Context-Aware Learning.

\section{Introduction}

In recent years there has been a technological explosion that has flooded our society with a wide range of different devices [1]. Moreover, the processing and storage capacity of these devices, their user interfaces or their communication skills are improved day by day. Thanks to these advances, we are currently surrounded by technology that has changed our habits and customs [2]. All this has caused the apparition of new fields such as Ambient Intelligence, whose main objective is to simplify the use of technology to improve people's quality of life [3].

Education is one of the areas in which Ambient Intelligence presents a greater potential as it provides new ways of interaction and communication between individuals and technological systems [4]. The usage of Information and Communication Technologies (ICT) has been present in educational innovations over recent years [4], modernizing the traditional transmission of contents through electronic presentations, email or more complex learning platforms such as Moodle or LAMS and fostering collaboration between students (Collaborative Learning) [5]. Beside the use of those general-purpose tools in education, other tools that make more specific use of 
technology have appeared. This applies to those that make use of Context-awareness information and ubiquitous computing and communication, fundamental parts of Ambient Intelligence [6].

The inclusion of context-awareness in educational scenarios and processes refers to Context-aware Learning [7], a particular area of application of Context-aware Computing [8]. Moreover, the ability to characterize and customize the context that surrounds a learning situation at a certain time and place provides flexibility in the educational process. This way, learning does not only occur in classrooms, but also in a museum, park or any other place [9], obtaining ubiquitous learning spaces. Thus, there is an extensive literature that addresses the problem of this kind of learning, highlighting those works that attempt to solve contextual information acquisition and providing data to users [9]. The use and integration of different technologies and the approach to specific learning activities characterize these solutions. However, the complexity of understanding and use of the technology and solutions in the aforementioned works does not allow a wide use of them.

This paper presents CAFCLA, a framework aimed at designing, developing and deploying AmI-based educational scenarios. Teachers are able to characterize the context where the learning activity will occur through the creation of a world model in which locate data collectors (e.g., sensors), identify and characterize areas of interest (e.g., paintings in a museum), etc. Moreover, the collaboration between students and the customization of the information available is also provided and can be integrated in the activity design. The framework is supported by a multi-agent architecture that provides intelligence to the learning process by helping to manage the activity, all the communications involved, the context-awareness and the collaboration between students and teachers. In addition, developers and technicians benefit from the Application Programming Interface and the formal schemas provided by CAFCLA.

The following section describes the background and problem description related to the presented approach. Then, the main characteristics of the context-aware technologies that CAFCLA integrates are described. Finally, the conclusions and future work are depicted.

\section{Background and Problem Description}

Providing contextual information and fostering collaboration between students benefit the learning process [1]. Moreover the combination of Collaborative and Contextaware Learning naturally leads to thinking about ubiquitous learning spaces, characterized by "providing intuitive ways for identifying right collaborators, right contents and right services in the right place at the right time based on learners surrounding context such as where and when the learners are (time and space), what the learning resources and services available for the learners, and who are the learning collaborators that match the learners' needs" [11].

A better understanding of environment through technology allows educators to customize the content provided to students. Similarly, technology facilitates the interaction with the environment and between students. This should be reached in a way as transparent and ubiquitous as possible. The technologies used for the collection of contextual information and for the communication between different devices are the 
cornerstone of the different works presented here. Literature about Context-aware Learning proposals has been deeply reviewed in this work. Some of the most representative works are classified in this paper, following technological criteria related to communications and data collection.

A first approach to provide contextual information is "tagging the context". Even though RFID (Radio Frequency IDentification) is the most spread technology [14], there are other technologies such as NFC (Near Field Communication) or QR Codes (Quick Response Codes) [12] which are growing fast. As can be seen in the usage of Active RFID, both location and context-awareness are closely related: knowing precisely location of objects and people allows determining what is surrounding them and, consequently, characterizing the context in which they are involved. GPS (Global Positioning System) is the most used technology to provide location in Contextaware Learning [13]. This location system provides a high accuracy level and is currently implemented in a wide range of smart phones and mobile devices. In those cases, the mobile device provides a position to the system. However, most of those works do not implement a specific case of use, but propose a general purpose model in which GPS technology is included to facilitate the provision of contextual data.

Furthermore, GPS technology does not work indoors because of the direct vision necessary between satellites and devices. However, indoor environments are very common in learning: museums, laboratories or the school are places where activities that require mobility can be developed. Trying to cover this lack, different location systems based on Active RFID [14] or Wi-Fi [10] are used. Both cases the performance of systems is similar: student's position is determined by the access point which is providing coverage in each moment. This type of approach has significant limitations when developing context-aware learning activities: the location accuracy is too poor. This situation presents an important problem when areas where context information is different are close (e.g., two paintings in a museum).

The review of the literature evidences some lacks in the Context-aware Learning systems proposed until now. Even some works try to combine different technologies to cover as much situations as possible [10], most of them only cover specific learning situations, as those where tagging context with RFID/NFC [12] is necessary or those where learning occurs outdoors [13]. The combination of both situations is only addressed by M2learn [10]. However, this solution does not provide a precise and efficient location systems or the possibility to integrate wireless sensor networks, except for RFID systems.

However, none of the solutions mentioned before takes into account Ambient Intelligence guidelines. The proposed solutions focus their work on the architectural description, framework developers or end-user applications whose designers have not taken into account how complex will be them for educators or students. Some aspects such as designing intuitive and attractive interfaces or abstracting end users from the complexity of technology, issues on which Ambient Intelligence pays special attention, are not taken into account. Thus, if these aspects are excluded from each solution's design process, the final result may be rejected by students and educators. For this reason, the design process must take into account, from the beginning, the opinion of all the interested parties [5], that is, educators, designers and developers. This way is easier to accomplish with Ambient Intelligent issues related to user interfaces and usability of final applications. 
Moreover, the works analyzed in this review do not include mechanisms for data or communication management. Ambient Intelligence emphasizes the transparency of technologies for users. In addition, technology is used to ease ordinary tasks or improve activities and the quality of life [6]. In this sense, systems that combine different technologies do not facilitate mechanisms to change between them (e.g., different communication protocols) attending to the needs of a situation. Similarly, data have to be managed in an intelligent and efficient way. Most of the literature reviewed does not include this issue, using only standard data repositories that only consider persistency and consistency [1]. Functionalities like data redundancy to solve network failures help to make the system dynamic and benefit data accessibility with independence of the place and the moment.

Even though it is well known that collaboration benefits the learning process [1], collaboration between students is an issue not considered by many proposals [10]. Including mobile devices and wireless communication protocols in any learning design that requires mobility (as discussed in this paper) is nowadays necessary. Mobile devices easily connect each other so including collaboration between students is an easy task, increasing the variety of activities and improving the learning process.

Furthermore, this work is developed following Ambient Intelligence guidelines, such as personalization of the provided context or transparency and ease of use for teachers and users. Moreover, the inclusion of reasoning mechanisms facilitate the personalization of data provision or the communication management of these kinds of complex systems [2].

\section{CAFCLA Context-Aware Technologies Integration}

CAFCLA is a framework aimed at designing, developing and deploying AmI-based educational scenarios, focusing on collaborative and context-aware activities. The framework integrates a set of wireless context-aware technologies and communication protocols (e.g., GPS, ZigBee, Wi-Fi, or GPRS/UMTS). Those technologies allow establishing collaborative activities based on Ambient Intelligence among students and teachers. In this sense, communication models vary dynamically depending on the activity; for example, following a client-server model to perform a data query or forming an ad-hoc network to gather contextual information. Thus, the contextual information is always available and may be modified every time. Contextual information is useful in the educational process, facilitating the acquisition of new knowledge and training. The use of contextual information allows a better understanding of the environment surrounding the learning and student in a given time. With this knowledge, the information received by the students can be dynamically optimized, customized and adapted to their needs and requirements. In order to provide contextual information for a wide range of learning scenarios, CAFCLA integrates three different context-aware technologies. More specifically, CAFCLA integrates indoor and outdoor location capabilities and a platform to deploy wireless sensor networks.

As shown in Figure 1, the main purpose of integrating these technologies is to cover the widest range of possible learning situations. Thus, the contextualization of any object or place will be facilitated by an outside location and / or a representative indoor plan of the place or places where the learning activity is taking place. Thus, the 


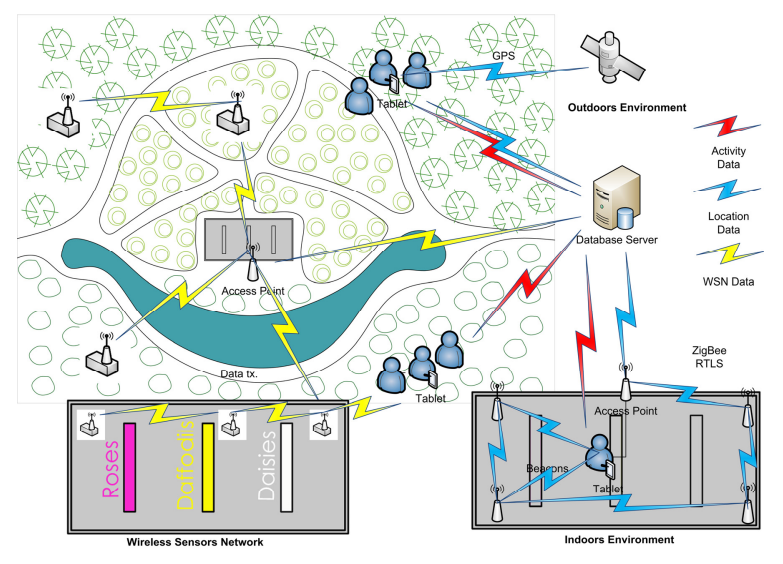

Fig. 1. CAFCLA working schema

system knows when and what information should be provided to students based on knowledge of their positions. Moreover, some contextualization can involve physical measurements, so CAFCLA offers the deployment of wireless sensor networks.

The functionalities of each of the context sensitive technologies integrated into the framework are described in the next sections.

\subsection{Wireless Sensor Networks}

The integration of a platform to deploy sensor networks in the framework is useful to cover situations in which the contextualization of an environment requires the collection of physical quantities. CAFCLA integrates the n-Core platform [15], which allows the integration of multiple sensors (e.g., temperature, humidity or pressure). The sensors form a mesh network through which data are sent to the access point that sends information to the CAFCLA data server. There, information is stored and processed. Moreover, the sensors can be connected with other ZigBee devices (e.g., a laptop or Tablet PC) and share the data they collect through an ad-hoc connection established in that moment for that purpose. In this case, educators must decide the location of each sensor, the data type and how often data have to be collected and sent to CAFCLA data server. So CAFCLA records where each sensor is placed and implements the protocol to communicate with other sensors, users or data server. Students will be able to receive data from sensors as they approach them by forming an ad-hoc network. Furthermore, the system is aware of which student has approached, so that the information provided can be customized or filtered.

\subsection{Outdoor Real Time Locating System}

The outdoor location system, specifically the GPS positioning system, is fully integrated into a wide range of mobile devices. For this reason, it is easy to integrate this technology into learning activities. This functionality requires a GPS device and maps platform like Google Maps or OpenStreetMap. CAFCLA integrates all the necessary logical background that may be available on the system and hides all the complexity 
inherent to the use of this technology to educators. When designing an activity, teachers draw an area on the map. There, all the contextual information related to the area is placed, including different versions of information that are used in different activities or by different users. The system is capable of associating an area to one or more descriptions, so that personalization is easy to achieve. During the development of the activity, students use an integrated GPS device that transmits its position continuously. When the student enters into a characterized area or approaches an object of interest, he or she receives contextual information according to the design of the activity.

\subsection{Indoor Real Time Locating System}

CAFCLA also provides an indoor Real Time Locating System. The main reason to include this technology is the technical failure of the GPS system to determine the position of users indoors. The Real Time Locating System is based on n-Core Polaris [15], a system that uses the ZigBee wireless communication protocol and that determines the position of users with up to 1 meter accuracy. The n-Core platform facilitates the localization process. The area where the tracking system is deployed is equipped with a set of beacons called n-Core Sirius D. These beacons are able to communicate and send information about the location of a student to the network access point. Each student has a ZigBee device called n-Core Quantum that communicates with the beacons closest to his position. Beacons collect different types of signals sent by mobile devices and send them to the access point. The access point sends all information to the activity server where a location engine calculates the position of the student. Teachers include any kind of information related to any area and students can receive the same way as it is done with the GPS tracking system. Thus, the complexity for educators is reduced and they only have to worry about what information is included in the system regardless of where they perform contextualization.

\section{Conclusions and Future Work}

The use of Information and Communication Technology in the different areas has increased in recent years thanks to the emergence in society of mobile devices, easy access to currently existing technology and the many features they present, such as communication protocols and context-aware technologies. However, it is difficult to develop applications to squeeze all the potential offered by technology, especially when the main objective is the development of technological applications that are transparent to users, as is suggested by the paradigm of Ambient Intelligence. CAFCLA is a framework designed within the education field with the objective to design and develop a set of tools that provide a basis for designing, developing and implementing Ambient Intelligence based collaborative learning activities that use contextual information. CAFCLA is a framework that integrates different contextaware technologies, such as Real Time Locating Systems, and several communication protocols that abstract educators and developers of context-aware collaborative learning activities from the complexity of the use of different technologies simultaneously. In this case, CAFCLA focuses on provide a set of tools and methods to teachers, developers and technical staff in order to easily design, develop and deploy this type of 
learning activities. CAFCLA has been designed following the guidelines established by AmI. Requirements such as adaptation, context awareness, anticipation or reasoning have been covered by the implementation of different context-aware technologies that allow the framework to be able to cover a wide range of learning scenarios. Moreover, CAFCLA presents an innovative way to design and develop learning activities, taking into account all staff involved in the process, facilitating the tasks each one is responsible. Future work includes the design, development and deployment of a specific use case where all the features of CAFCLA are implemented. This work will be developed by different teachers and developers in order to compare the results reached by all of them and evaluate the framework in different real scenarios. So that, all the features implemented by CAFCLA will be evaluated and improved thanks to the feedback of final users.

Acknowledgments. This work is supported by the Spanish Ministry of economy and competitiveness.

\section{References}

[1] García, Ó., Tapia, D.I., Alonso, R.S., Rodríguez, S., Corchado, J.M.: Ambient intelligence and collaborative e-learning: a new definition model. Journal of Ambient Intelligence and Humanized Computing, 1-9 (2011)

[2] Jorrín-Abellán, I.M., Stake, R.E.: Does Ubiquitous Learning Call for Ubiquitous Forms of Formal Evaluation?: An Evaluand oriented Responsive Evaluation Model. In: Ubiquitous Learning: An International Journal. Common Ground Publisher, Melbourne (2009)

[3] Tapia, D.I., Abraham, A., Corchado, J.M., Alonso, R.S.: Agents and ambient intelligence: case studies. Journal of Ambient Intelligence and Humanized Computing 1(2), 85-93 (2009)

[4] Scardamalia, M., Bereiter, C., McLean, R.S., Swallow, J., Woodruff, E.: ComputerSupported Intentional Learning Environments. Journal of Educational Computing Research 5(1), 51-68 (1989)

[5] Gómez-Sánchez, E., Bote-Lorenzo, M.L., Jorrín-Abellán, I.M., Vega-Gorgojo, G., Asensio-Pérez, J.I., Dimitriadis, Y.A.: Conceptual framework for design, technological support and evaluation of collaborative learning. International Journal of Engineering Education 25(3), 557-568 (2009)

[6] Traynor, D., Xie, E., Curran, K.: Context-Awareness in Ambient Intelligence. International Journal of Ambient Computing and Intelligence 2(1), 13-23 (2010)

[7] Laine, T.H., Joy, M.S.: Survey on Context-Aware Pervasive Learning Environments. International Journal of Interactive Mobile Technologies 3(1), 70-76 (2009)

[8] Dey, A.K.: Understanding and Using Context. Personal and Ubiquitous Computing 5(1), 4-7 (2001)

[9] Bruce, B.C.: Ubiquitous learning, ubiquitous computing, and lived experience. In: Cope, W., Kalantzis, M. (eds.) Ubiquitous Learning, pp. 21-30. University of Illinois Press, Champaign (2008)

[10] Martín, S., Peire, J., Castro, M.: M2Learn: Towards a homogeneous vision of advanced mobile learning development. In: 2010 IEEE Education Engineering (EDUCON), pp. 569-574 (2010) 
[11] Hwang, G.-J., Yang, T.-C., Tsai, C.-C., Yang, S.J.H.: A context-aware ubiquitous learning environment for conducting complex science experiments. Computers \& Education 53(2), 402-413 (2009)

[12] Tan, Q., Kinshuk, Kuo, Y.-H., Jeng, Y.-L., Wu, P.-H., Huang, Y.-M., Liu, T.-C., et al.: Location-Based Adaptive Mobile Learning Research Framework and Topics. In: International Conference on Computational Science and Engineering, CSE 2009, vol. 1, pp. 140-147 (2009)

[13] Driver, C., Clarke, S.: An application framework for mobile, context-aware trails. Pervasive and Mobile Computing 4(5), 719-736 (2008)

[14] Blöckner, M., Danti, S., Forrai, J., Broll, G., De Luca, A.: Please touch the exhibits!: using NFC-based interaction for exploring a museum. In: Proceedings of the 11th International Conference on Human-Computer Interaction with Mobile Devices and Services, MobileHCI 2009, vol. 71, pp. 1-2 (2009)

[15] Nebusens, n-Core ${ }^{\circledR}:$ A Faster and Easier Way to Create Wireless Sensor Networks. n-Core® (2012), http: / / www . n-core. info (last access: January 16, 2013) 